\title{
Vision-based drowsiness detector for a Realistic Driving Simulator
}

\author{
I. García, S. Bronte, L. M. Bergasa, \\ N. Hernandez \\ Departament of Electronics \\ University of Alcalá \\ Madrid, Spain \\ \{ivan.garcia,sebastian.bronte,bergasa $\}$ \\ @ depeca.uah.es
}

\author{
B. Delgado, M. Sevillano \\ Research and Project Department, ESM \\ Asturias, Spain \\ \{beatriz,matiassevillano\}@esm.es
}

\begin{abstract}
This paper presents a nonintrusive approach for monitoring driver drowsiness, based on computer vision techniques, installed in a realistic driving simulator. An IR stereo camera is placed in from of the driver in order to obtain PERCLOS, the most confident drowsiness parameter [1], in real-time and in a robust and automatic way. Our proposal doesn't need a calibration process and includes three main stages. The first is the pre-processing stage, which includes face and eye detection based on appearance strategy using the Viola and Jones algorithm, and the equalization of the eyes using a Hat transformation. An eye tracking strategy in a sequence of image frames is then carried out. The second stage executes the pupil position extraction and its characterization using integral projection techniques and a Gaussian model. The final stage executes the PERCLOS estimation, depending on the eyes closed rate on duration of time interval and fusing information obtained for each eye in the two images of the stereo camera. For evaluation of the proposed system several experiments have been designed by psychologists and carried out. A preliminary study about the performance of the proposal, based on confusion matrixes, is presented.
\end{abstract}

Index Terms-Intelligent Transportation Systems, Driver Drowsiness, Confusion Matrix, PERcentage of eye CLOSure (PERCLOS), Visual Fatigue Behaviour.

\section{INTRODUCTION}

Sleepiness during driving has been shown to result in a greatly increased risk of suffering an accident. Specifically, Klauer et al. [2] has shown that to drive while sleepy increases the accident risk four to six times, compared to alert driving. Furthermore, the risk of suffering an accident is higher during night driving [3] or in situations with reduced prior sleep [4]. In fact, at least $15-20 \%$ of all vehicle accidents have been estimated to be sleepiness related [5]. Therefore, it is beneficial to develop a system to monitor the physical and mental state of the driver and give alerts at the critical moment when the driver is becoming fatigued, thereby preventing traffic accidents.

In the last decade, diverse techniques have been used to develop monitoring systems for a variety of purposes. Those techniques used to detect a driver's sleepiness can be generally divided into three main categories [1]. The first category includes methods based on biomedical signals, like cerebral, muscular and cardiovascular activity [6], [7] and [8]. Usually, these methods require electrodes attached to the driver's body, which will often cause annoyance to the driver Most of them are yet far from being effectively introduced in the market, according to recent reviews [9].

The second category includes methods based on driver performance, which evaluate variations in the lateral position of the vehicle, in the velocity, in the steering wheel angle and in other signals recorded by CAN [10], [11] and [12] The advantage of these approaches is that the signal is meaningful and the signal acquisition is quite easy. This is the reason because such systems have indeed entered the commercial market [13], [14] and [15] but, to the author's knowledge, in the open literature there are very few details available regarding the mechanisms or parameters of these systems. On the other hand, these systems are subject to several limitations such as vehicle type, driver experience, geometric characteristics, condition of the road, etc. Then, these procedures require a considerable amount of time to analyze user behaviours and therefore, they do not work with the so called micro-sleeps-when a drowsy driver falls asleep for a few seconds on a very straight road section without changing the vehicle signals.

The third category includes methods based on driver visual analysis using image processing techniques. Computer vision can be a natural and nonintrusive technique for monitoring driver's sleepiness from the images taken by some cameras placed in front of the user. These approaches are effective because of the occurrence of sleepiness is reflected through the driver's face appearance and head/eyes activity. Different kinds of cameras and analysis algorithms have been reported in the literature for this approach: methods based on visible spectrum camera [16] and [17]; methods based on IR camera [1], [18], [19] and [20]; and methods based on stereo camera [21] and [22]. Some of them are commercial products as: Smart Eye [19], Seeing Machines DSS [20], Smart Eye Pro [21] and Seeing Machines Face API [22]. However, these commercial products are still limited to some well controlled environments and they require of hard calibration processes. Then, there is still a long way in order to obtain a robust commercial product in this category.

Regardless of the type of measurement, one of the chief problems of drowsiness detection studies is the difficulty of carrying out experimental tests to validate the techniques. 
These tests are often conducted in laboratory with driving simulators, because for safety reasons, road tests in real vehicles have strong limitations. Laboratory settings allow using controlled environments, in which drowsiness can be induced, and it is possible to use many measurement equipments that are difficult to integrate in real vehicles. However, the principal limitations of laboratory experiments are their lower realism and the risk of simulator sickness [23]. Another important problem, in both road and laboratory studies, is the alteration of the spontaneous behaviour of drivers: drowsiness in real driving is caused by a combination of the accumulated fatigue of the driver and the tiresomeness of a monotonous task, specially in familiar roads and vehicles. The unusual experience of participating in such an experiment, specially if there is an invasive instrumentation, or the "white coat effect" due to the presence of researchers, may hinder drowsiness; or on the contrary, the higher level of stimulation in real road conditions may reduce sleepiness [24]. This limits the efficacy of the experiments, since to validate the models of drowsiness detection, it is important to have a balanced and realistic quantity of records of users in both wakeful and drowsy periods.

This paper presents a nonintrusive approach for monitoring driver drowsiness, based on computer vision techniques, installed on a realistic driving simulator. An IR stereo camera is placed in front of the driver in order to obtain PERCLOS, the most confident drowsiness parameter [1], in real-time and in a robust and automatic way. Our proposal doesn't need a calibration process and includes techniques to overcome the typical problems of image processing algorithms as are: lighting conditions changes, user appearance and quick movements. For evaluation of the proposed system several experiments have been designed by psychologists and carried out with a twofold objective: (a) To gather a database of driving performance parameters, obtained from the simulator, and PERCLOS, from professional drivers in both wakeful and drowsy conditions, which may be successfully used to study the measurable changes related to fall of attention and drowsiness and accordantly to find patterns in those signals. And (b) to validate correlation between subjective sleepiness method used in this work as ground-truth, and PERCLOS objective measures obtained with our system.

In section II the simulator, the method used to study the fatigue and drowsiness in drivers and the generation of the ground truth signal are described. Also, in section III the algorithm used to detect the drowsiness or PERCLOS in drivers is explained. After that, results associated with one video sequence and its comparison with the ground truth is presented in IV. Finally in section V conclusions and future works are presented.

\section{EXPERIMENTS AND DATA COLLECTION}

The purpose of this section is to show the characteristics of a realistic simulator and to explain the methodology carried out by psychologists in the analysis task to evaluate the drowsiness in professional drivers.

\section{A. Realistic driving simulator}

Simulation methodologies applied to training and research in the field of road transport in Europe, have been proved to be both cost-effective and efficient. Simulation aims to give the driver an opportunity to immerse himself in his habitual workplace. The study employed the realistic generation highfidelity simulator, shown in Fig. 1, at the CEIT [25].

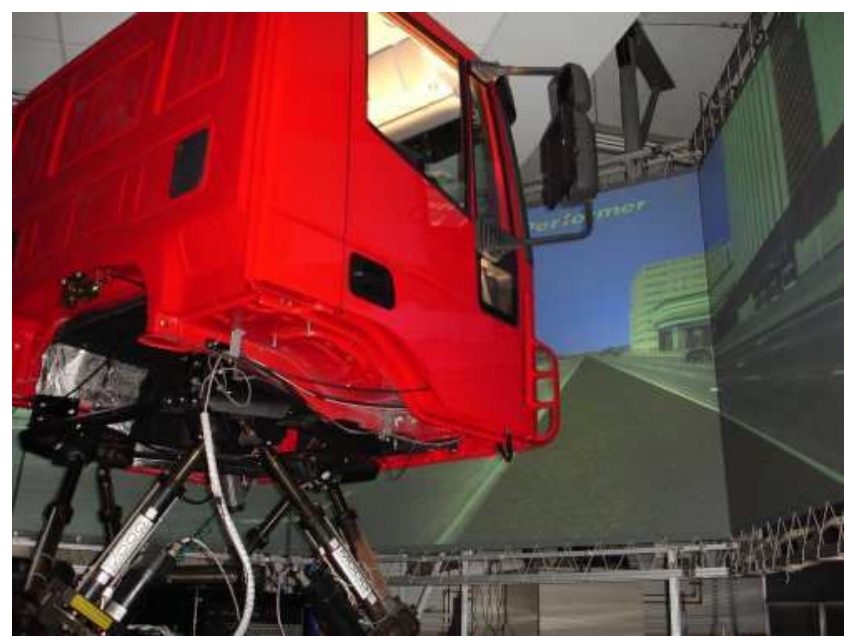

Fig. 1. Simulator

The truck simulator has a real truck cab, an instructor station, a rack of computers and a system of screens that cover 180 degrees of vision. The truck cab is an Iveco Stralis cab mounted on a Stewart platform 6GDL MOOG Company. The electronics in the cabin have been replaced by new electronics designed by CEIT. It has sensorised the steering wheel with switches, levers and pedals and changed the instrument panel by a virtual panel that allows configuration to measure the different aspects of information to be displayed to the driver. This is integrated into the cockpit of the simulator. The simulator has 3 scenarios (urban, interurban and mountain) with more than $250 \mathrm{~km}$ of roads allow driving in adverse conditions rain or snow, and at different times of day or night. For the visualisation of the simulation a set of 3 screens with re-configurable positions cover a large area showing all angles of vision of drivers. Two further monitors act as mirrors to the truck. Thus, this simulator gives the researcher great flexibility to implement recording systems specifically designed to test the reactions and behaviour of professional drivers while they are driving [26].

Psychologists need the simulator to have installed specific systems to register the professional drivers behaviour. For this propose, the realistic simulator register driver and driving variables, wich the following systems:

- A stereo vision system based on active infrared lighting to record the driver's face.

- A recording system for the identification of parameters related to driving behaviour (vehicle speed, steeringwheel movement analysis, specific devices for the quan- 
tification of the driver's condition of alertness, etc.).

The driver drownsiness signal is accurate because wide experienced phycologist studied the registered variables.

The extreme driving conditions to which the drivers must be exposed make it so that, only through a full-immersion simulated environment, it is possible to develop the necessary technology for the identification, detection and warning of a state of drowsiness while driving.

\section{B. Experimental protocol}

The main target of this experimental protocol is to recreate a suitable environment which would enable researchers to detect drowsiness during driving. A large amount of information about drowsiness detection studies and projects also has been gathered and, lastly, we have wide experience in extensive research into the professional driving field. The driving task has been suitably studied by experts in Human Factors. Their work allows formulating working hypothesis which become part of the wording of the different simulation exercises created with a view to developing and optimising detection systems of drowsiness [27].

The data were collected from a simulator study in which a total of 20 test subjects participated. The study was designed such that each test subject would carry out driving sessions during two different conditions: Either after having slept on a regular schedule (from 23:00 to 07:00, with allowance for one hour deviations from the schedule) for two nights prior to the day of the experiment, or after having slept only four hours during the night preceding the experiment, thus being partially sleep deprived. Each test subject carried out six driving sessions under each of the two conditions, spread over a 24-hour period. Each driving session lasted 60 minutes. The tests subjects that participated in the study were recruited from the Spanish national register of vehicle owners, by random selection (only private vehicle owners were included). They had to be frequent drivers, driving at least $5000 \mathrm{~km}$ a year, and not suffer from habitual sleep disturbances.

The problem of sleepiness detection has been usually treated as a binary classification problem. In this work we propose to use three classes in order to have a finer classification of the driver's state taking into account that the Karolinska Sleepiness Scale (KSS) [28] has 9 different levels. Then, to evaluate performance of our detector, the problem of sleepiness detection was cast as a three level classification problem, in which the data collected during a given period of time (60 seconds, here referred to as a driving period) was considered as having originated from an alert driver, a fatigued driver or a sleepy driver. The class assignment of the data from a given driving period was based on the subjective sleepiness estimation of three annotators. Taking the more repetitive estimations of the experts as ground truth signal, the objective of the detector was to classify correctly as many driving periods as possible. Experts were trained to estimate driver's levels of sleepiness in an off-line process analysing video sequences and driving signals performance obtained for each user during the driving sessions. The estimates were based on the KSS that ranges, in integer steps, from 1 (extremely alert) to 9 (very sleepy, fighting sleep). However, in order to simplify the evaluation and due to we have only three different categories in the classification, only three classes where required for the expert estimations (awake, fatigued, drownsiness). The Karolisca scale is closely related to EEG and behavioral variables, indicating a high validity in measuring sleepiness.

Experts can make off-line studies to determinate the driver drowsiness state every minute with the recoded driver and driving information and the proposal scale to evaluate the drowsiness. While subjective estimations of sleepiness are commonly used in studies on driver sleepiness, some researchers have also considered objective measures of sleepiness, in some cases also making comparisons with subjective measures. To use subjective estimations of sleepiness as ground truth is accepted for some authors but is rejected for some others, being an open point in this discipline. We have used this option because we don't want to disturb the spontaneous behaviour of drivers due to invasive instrumentation or the presence of experts in the test. Then, our proposal is to use subjective estimations obtained in an off-line process analysing video sequences and driving signals performance obtained for each user during the driving sessions. Additionally, one of our goals in this work is to analyse correlation between subjective estimations of experts and an objective measures as is PERCLOS.

\section{ALGORITHM FOR VISUAL-BASED DROWSINESS DETECTION}

The main system architecture diagram is shown in Fig. 2. The input image, which is taken from an infrared sensitive camera and active illumination, corresponds to a driver face who can be affected by different states of drowsiness. The input image is processed to detect face and eyes applying Viola and Jones algorithm and a Kalman filter to smooth position variation. Once the eyes are detected, iris centre location algorithm is executed. It is based on integral projections and then a Gaussian model is correlated to obtain eye centre and opening. This last parameter will be used to measure PERCLOS. The PERCLOS is calculated in the two image supplied by the stereo camera system and then the mean of both values are evaluated. The comparison between PERCLOS signal obtained from the system and the ground truth generated by experts will show the accuracy of the drowsiness detection algorithm.

\section{A. Face and Eye detection}

In this step eyes position will be located throughout the whole image sequence. In general, it is very complicated because of the different object colours, expressions, poses, relative sizes and very uneven illumination conditions.

From all the techniques that are related to pattern recognition, particularly in face and eye detection, Viola \& Jones [29] detector is selected because while the driver face is in frontal orientation the Viola \& Jones detection rate is very hight and robust the PERCLOS measure. When the face 


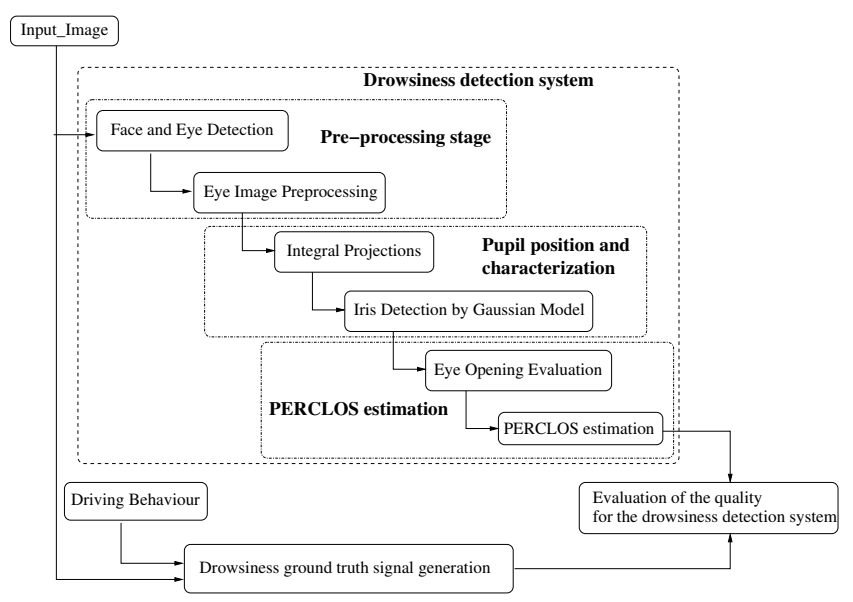

Fig. 2. General diagram

orientation is different the PERCLOS value is decremented. This statistical method does not need any kind of previous information from a concrete driver face. The information needed to perform an object detection is extracted from the training samples base.

This algorithm consist on a vector of Haar features extracted from input images which are the input of cascade classifiers. These are hierarchical classifiers put one after another one shorted by the importance of the features. The direct application of this algorithm over the whole image does not give good results. Because of that, eye search is made in two steps. The first step is to look for the face on the image, and once the area is found, eye detector is applied on it. Detection failures in consecutive images are fixed with a Kalman filter [18] per eye, and it keeps tracking with the prediction only for a few frames. The Kalman filter model is used to robustness the PERCLOS meausere in view of quick face movements.

Face and eyes' detection regions are shown in Fig. 3.

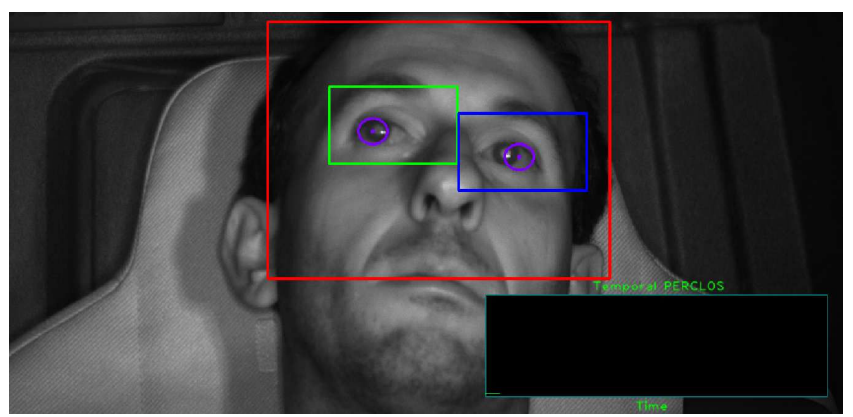

Fig. 3. Face and eyes detection

\section{B. Eye Image Preprocessing}

Once the region of interest is defined for each eye with Viola \& Jones, a hat transformation is applied. This transformation consists of the subtraction from the original image to the image in which a closing operation is applied. This transformation erases most of the bright little parts inside the eye and smoother images are obtained. To obtain more robustness against illumination variations, an uniform equalization is then used. In Fig. 4 three different images are shown. The first one is the original image detected by Viola and Jones, the second one is the result of the hat transformation, and the third one is the equalised image.

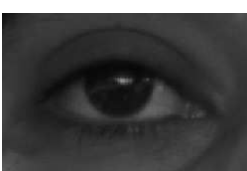

(a)

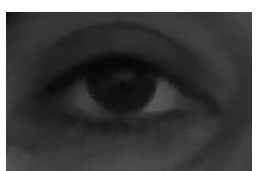

(b)

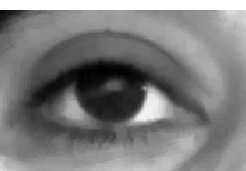

(c)
Fig. 4. (a) Original eye detection; (b) Hat transform; (c) Equalized image

Eye features can be easily analyzed in the equalized image than in the original one. The objetive of this stage is to improve the image quality to calculate the eyes height and width needed to detect driver drowsiness in a correct way and robustness to lighting condition changes.

\section{Integral projections}

The concept of integral projection of an image assumes that vertical projection is the average of the pixels from each column, horizontal projection is the average of the pixels from each row. It could be also done for any angle, taking the average of the pixels in a specific direction.

Given an image $I$ with dimensions $W x H$ the integral projections (horizontal $P_{H}$ and vertical $P_{V}$ ) are defined by the following equations:

$$
\begin{aligned}
& P_{H}(y)=\frac{1}{W} \cdot \sum_{x=0}^{W-1} I(x, y) ; \forall y=0, \ldots, H-1 \\
& P_{V}(x)=\frac{1}{H} \cdot \sum_{y=0}^{H-1} I(x, y) ; \forall x=0, \ldots, W-1
\end{aligned}
$$

The eye image has a circular gray level distribution due to the iris features. These caracteristics do that the projective integral in both axes has a Gaussian form with different mode, median and mean parameters. For this reasson a Gaussian model is used to evaluate the iris features.

\section{Gaussian model}

The Gaussian model is characterized by (3). This equation has two parameters that determines the typical open eye shape.

$$
f(x)=\frac{1}{\sqrt{2 \pi \sigma^{2}}} e^{\frac{-(x-\mu)^{2}}{2 \sigma^{2}}}
$$

In Fig. 5 the results of applying integral projections to an eye are shown. The iris centre estimation is obtained convolving Gaussian functions with the projected arrays. The maximum obtained from the convolution with the Gaussian template corresponds with the most likely coordinate in which the iris centre can be found. 
Because some times this maximun does not correspond with the iris centre, an area near to this point is evaluated, measuring the mode and the median parameters. When density distributions are gaussian, unimodal, and reasonably asymmetric. To measure asymmetry, Pearson coefficient is calculated as follows in (4).

$$
A_{p}=\frac{\text { median }- \text { mode }}{\text { standard deviation }}
$$

If the density distribution is symmetric, the following condition is satisfied: median $=$ mode and $A_{p}=0$. On the contrary, if there is an asymmetric function, $A_{p}>0$. This is very important to assert if there is an eye or another dark object on the image. Experimentally projected functions are found to have quite symmetric shape, which means that the absolute value of the difference between median and the mode is not too high. As a consequence, a low threshold is the only value that is needed to know if the dark object present on the image is an eye or not. This assumption is valid for the most part of the candidates tested in different conditions. In Fig. 5.(a) an iris centre detection is shown, in Fig. 5.(b) represents a half closed eye and because it is located in the right corner of the eyebrow the detected point is classified as non-iris.

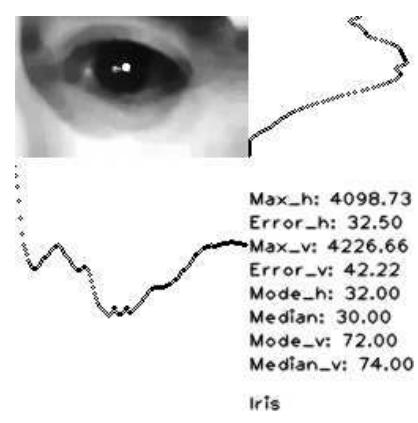

(a)

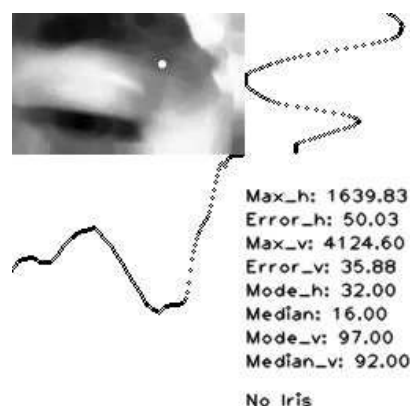

(b)
Fig. 5. Classification of iris and not iris

When driver's head is turned and the algorithm does not detect any iris the PERCLOS decreases due to this is a clue that the driver is awake.

\section{E. Eye opening evaluation}

Only when the algorithm detects an eye on the image, the eye opening is calculated. The eye opening or the iris height is evaluated measuring the standard deviation of the Gaussian that characterises the eye. In Fig. 6.a this concept is showed. To evaluate the eye opening percentage, a previous calibration process is needed. The calibration process is automatically made during the first 10 seconds of the exercise. The driver has to look to the road, when he is awake, in a relaxed position and the algorithm calculates the eye opening value.

With this value is possible to evaluate the instantaneous percentage of eye opening from the ratio between the iris height in the current frame and the one obtained in the calibration process. The eye opening percentage is used by the next process, which evaluate the PECLOS value. In Fig. 6.b the obtained results are shown.

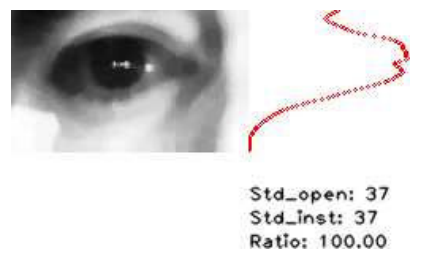

(a)

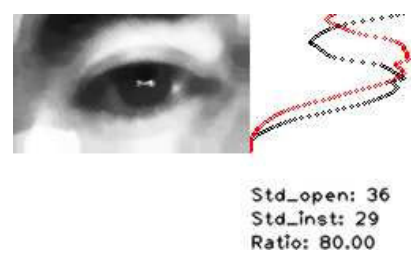

(b)
Fig. 6. Sample of aspect-ratio of the eye opening

\section{F. Drowsiness parameters estimation}

PERCLOS [30] is one of the most representative parameters when driver drowsiness is measured [31]. It shows how open and how long the eyes are opened computing the average in a temporal window, whose duration is commonly accepted to be 20 seconds.

To make this measurement more accurate, it is necessary to distinguish between blink and PERCLOS. Blinking time have to be removed from the accumulator to get better estimations. A state machine is designed to do that. The states are the following: open_eye, blink_eye and perclos_eye . Transitions between states are shown in Fig. 7.

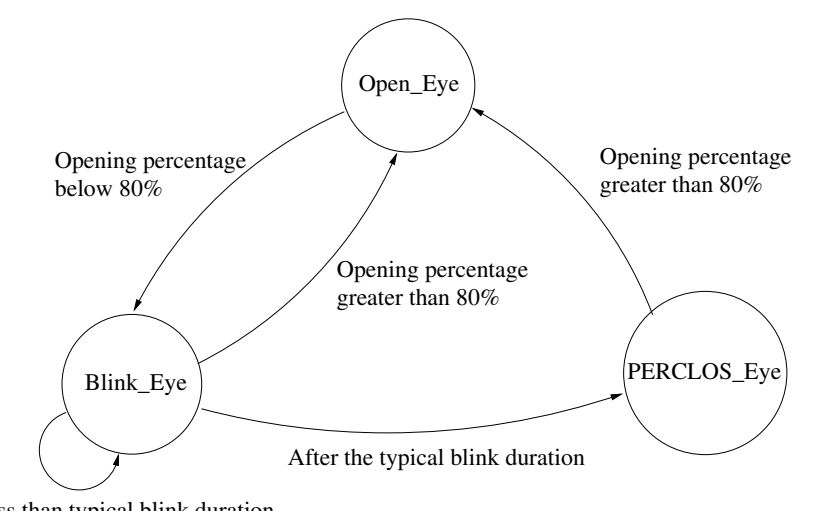

Less than typical blink duration

Fig. 7. State machine diagram of PERCLOS measurement

If eye opening is under a threshold, which is typically under the $80 \%$, it is assumed that the eye is closing and the next state will be blink_eye. If it rise, the next state will be open_eye. On the contrary if eye opening go under $20 \%$, two different cases can happen depending on the duration that the system keeps in this state. If duration is less than 3 frames there is no change, but else the state changes to perclos_eye when the duration is more than 3 frames under the $80 \%$.

Fig. 8 shows a temporal representation of the eye opening process. The PERCLOS calculates the variation of the eye opening during 20 seconds. 22 frames are showed in the figure. For each frame the eye is in a determined state. When the state machine is in the PERCLOS state the frame 
is marked with one and when the state is different to the PERCLOS the frame is marked with zero. This is shown on the PERCLOS evaluation windows in the Fig. 8. The average of the samples included in a temporal window of 20 seconds gives the PERCLOS value.

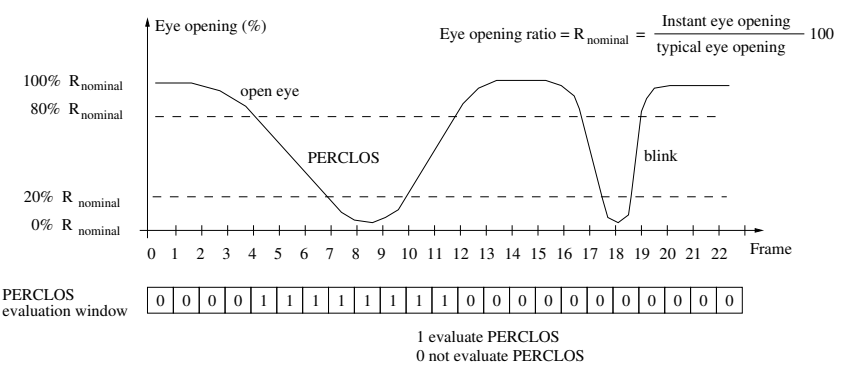

Fig. 8. Temporal tendency of eyes opening

PERCLOS is calculated in the two image supplied by the stereo system and then the mean of both values is taken as output value.

\section{EXPERIMENTAL RESULTS}

The results presented here are included within the CABINTEC project. The study was done with 20 users and the results obtained from one of them are shown. The scenario for this test has the following characteristics: a deprived user of sleeping and a duration about 85 minutes. The ground truth signal is assessed off-line by experts and it is used to evaluate PERCLOS signal accurately. Ground truth signal is shown at the top of Fig. 9. It is discretized into three levels: an initial awake level which is represented by 0 , a second fatigue level which is represented by 1 and a third drowsiness level which is represented by 2 .

PERCLOS is evaluated at a frequency of 30 times per second, recording about 144,000 PERCLOS measures throughout the test. Due to the ground truth signal is build every minute, the PERCLOS signal is decimated at a frequency of $1 \mathrm{~Hz}$ to compare both signals. The decimation is calculated using the average of the instantaneous values over a period of one minute. Once the PERCLOS value is calculated at this frequency, the signal is discretized into three levels, placing a threshold at $15 \%$ as the limit between wake and fatigue, and a threshold of $23 \%$ for the limit between fatigue and sleepiness, as shown in the graph below in Fig. 9. Once these thresholds are calculated under supervision by expert psychologists, the three-phases state system is obtained, as shown in the central graph of Fig. 9.

Due to the classifier is not binary, the typical ROC curves cannot be used. Paterl and Markey [32] proposed a method for the goodness of classifiers which have $\mathrm{N}$ different classes. In this case the confusion matrix will have $\mathrm{N}^{*}(\mathrm{~N}-1)$ elements and it is shown in the table I.

Where Awake(0) $\rightarrow \operatorname{Drow}(2)$ is the percentage of Awake(0) states that have been classified as Drow(2) ones.

The confusion matrix is calculated from the comparison between the ground truth and PERCLOS. Table II depicts the
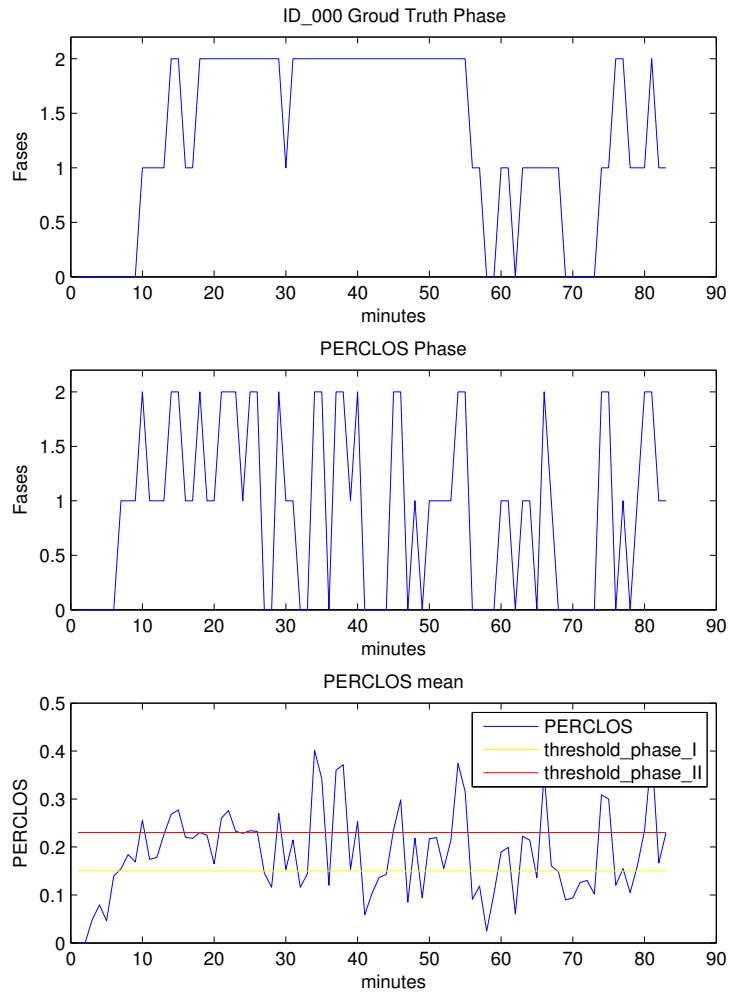

Fig. 9. Ground truth vs PERCLOS

TABLE I

CONFUSION MATRIX

\begin{tabular}{|c|c|c|c|}
\hline & Awake $_{G T}(0)$ & $F_{a t} t_{G T}(1)$ & $\operatorname{Drow}_{G T}(2)$ \\
\hline Awake $_{\text {det }}(0)$ & $\operatorname{Awake}(0) \rightarrow \operatorname{Awake}(0)$ & Fat $(1) \rightarrow$ Awake $(0)$ & $\operatorname{Drow}(2) \rightarrow \operatorname{Awake}(0)$ \\
\hline$F t_{d e t}(1)$ & $\operatorname{Awake}(0) \rightarrow \operatorname{Fat}(1)$ & Fat $(1) \rightarrow F a t(1)$ & $\operatorname{Drow}(2) \rightarrow \operatorname{Fat}(1)$ \\
\hline $\operatorname{Drow}_{\operatorname{det}}(2)$ & $\operatorname{Awake}(0) \rightarrow \operatorname{Drow}(2)$ & Fat $(1) \rightarrow \operatorname{Drow}(2)$ & $\operatorname{Drow}(2) \rightarrow \operatorname{Drow}(2)$ \\
\hline
\end{tabular}

confusion matrix obtained for one driver that present several drowsiness states.

TABLE II

CONFUSION MATRIX

\begin{tabular}{|c|c|c|c|}
\hline & Awake $_{G T}(0)$ & Fat $_{G T}(1)$ & $\operatorname{Drow}_{G T}(2)$ \\
\hline Awake $_{\text {det }}(0)$ & 0.8235 & 0.2083 & 0.2857 \\
\hline Fat $_{\text {det }}(1)$ & 0.1765 & 0.5833 & 0.2619 \\
\hline Drow $_{\text {det }}(2)$ & 0 & 0.2083 & 0.4524 \\
\hline
\end{tabular}

Once the confusion matrix is obtained an equilateral polygon of $\mathrm{N}^{*}(\mathrm{~N}-1)$ edges, percentages of bad classified results from the confusion matrix are represented on a radial graphic. In this case the shape will be a hexagon like the one shown in Fig. 10, which corresponds to the confusion matrix from table II.

The dark grey hexagon represents the random classification. The random classifier hexagon would have an inner hexagon if the clasification is good. The less area the hexagon has, the greater the specificity is and the better classifier is obtained. As it can be seen, the randon classification hexagon 


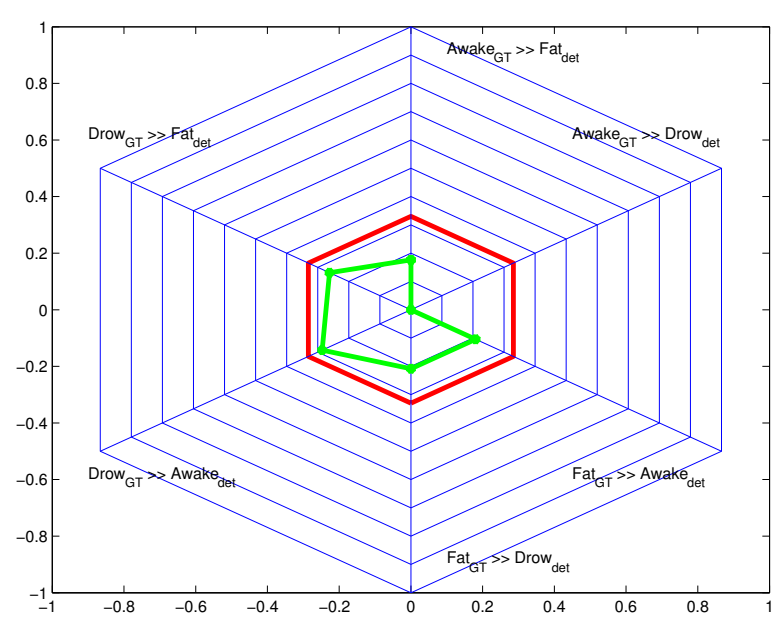

Fig. 10. Bad classification hexagon

contains the classifier hexagon, and it has a lower area than the random one.

Similarly, Fig. 11 represents a correct classification triangle, which corresponds with the confusion matrix from table II.

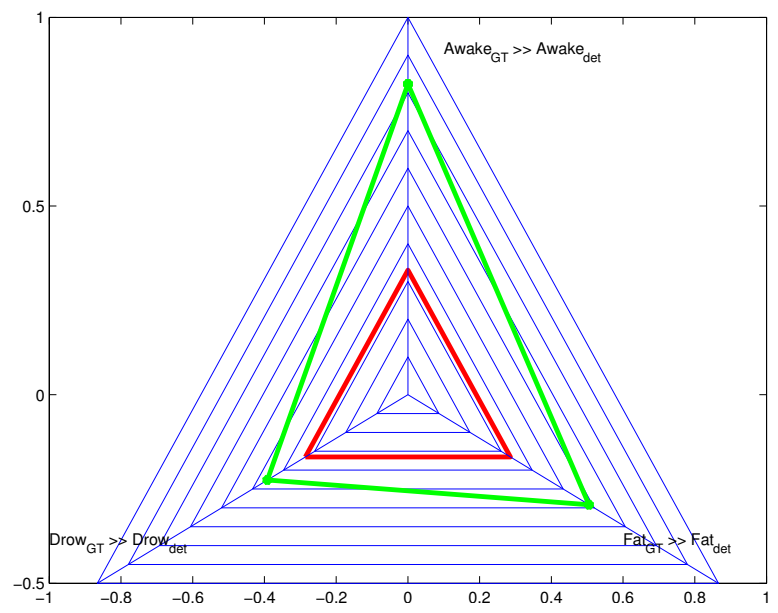

Fig. 11. Correct classification triangle

The dark grey triangle represents the random classifier. The random classifier triangle would have an outner triangle if the clasification is good. The greater area the triangle has, the greater the sensitivity is and the better classifier is obtained. It can be appreciated that the correct classification triangle contains the random classification triangle, and it has a greater area than the random one. The numeric resuls, in this concrete user, are $83 \%$ of awake state recall rate, $58 \%$ on fatigue state and $45 \%$ on drowsiness state.

In general, the mean results obtain by all users are $83.5 \%$ of awake state recall rate, $57 \%$ on fatigue state and $46 \%$ on drowsiness state. These results are similar to the user results showed.

\section{CONCLUSIONS AND FUTURE WORKS}

This paper presents a non intrusive approach for monitoring driver drowsiness, based on computer vision techniques, installed in a realistic driving simulator.

The proposed drowsiness detection method has demonstrated to be valid, showing an $85 \%$ of awake state recall rate considering only PERCLOS.

The computer vision and the Gaussian model method does not need fixed threshold to determine the eye opening. However the PERCLOS signal assessment needs a nominal or fixed eye opening threshold value. This nominal value is dependent on the constraints of the driver's eye and it is calculated in an initial automatic process.

The discrete drowsiness signal with three levels (awake, fatigue and drowsiness) resulting to the continuous PERCLOS signal is assessmented supervising the continuous PERCLOS signal with the users recorded videos in off-line mode. The annotators generate two thresholds that discretized the continuous signal in three levels. In future works, these two thresholds could be assessment with data analysis techniques and will be evaluated automatically. Introducing a neural classifier based on the first measures would be interesting to particularize it for each user.

Generate a ground truth drowsiness driver signal by expert psychologists is important to evaluate the recall rate of any method related with drowsiness detection.

The results shown in this paper are related to the described simulator. A good way to measure how robust is the algorithm presented is to apply it in outdoors conditions.

If a camera is removed, the system will be less expensive but will decrease the measure accuracy. The data fusion of the following parameters: PERCLOS, blink frequency, fixed gaze, lane deviation and amplitude of wheel turns are needed to increase the measure accuracy.

\section{ACKNOWLEDGMENTS}

This work has been financed with funds from the Ministerio de Ciencia e Innovación through the project DRIVERALERT (TRA2008 - 03600), as well as from the project CABINTEC (PSE-370000-2009-12).

\section{REFERENCES}

[1] L. M. Bergasa, J. Nuevo, M. Á. Sotelo, R. Barea, and M. E. L. Guillén, "Real-time system for monitoring driver vigilance," IEEE Transactions on Intelligent Transportation Systems, vol. 7, no. 1, pp. 63-77, 2006.

[2] S. G. Klauer, T. A. Dingus, V. L. Neale, , and J. D. Sudweeks, "The impact of driver inattention on near-crash/crash risk: An analysis using the 100-car naturalistic driving study data," National Highway Traffic Safety Administration, DC, DOT HS, vol. 810, 2006.

[3] T. Akerstedt, G. Kecklund, and L. Hörte, "Night driving, season, and the risk of highway accidents." Slee, vol. 24, pp. 401-406, 2001.

[4] J. Connor, R. Norton, S. Ameratunga, E. Robinson, I. Civil, R. Dunn, J. Bailey, and R. Jackson, "Driver sleepiness and risk of serious injury to car occupants: Population based control study." British Medical Journal, vol. 324, pp. 1125-1129, 2002.

[5] J. Horne and L. Reyner, "Vehicle accidents related to sleep: A review." Occupational and Environmental Medicine, vol. 56, pp. 189-294, 1999.

[6] T. Oron-Gilad, A. Ronen, and D. Shinar, "Alertness maintaining tasks (amts) while driving," Accident Analysis \& Prevention, vol. 40, no. 3, pp. 851-860, May 2008 . 
[7] C. Papadelis, Z. Chen, C. Kourtidou-Papadeli, P. Bamidis, I. Chouvarda, E. Bekiaris, and N. Maglaveras, "Monitoring sleepiness with on-board electrophysiological recordings for preventing sleep-deprived traffic accidents." Clinical Neurophysiology, vol. 118, no. 9, pp. 19061922, September 2007.

[8] J. Faber, "Detection of different levels of vigilance by eeg pseudo spectra." Neural Network World, vol. 14, no. 3-4, pp. 285-290, 2004.

[9] N. Wright, B. Stone, T. Horberry, and N. Reed, "A review of in-vehicle sleepiness detection devices," TRL Limited, Published Project Report PPR157, 2007.

[10] T. Wakita, K. Ozawa, C. Miyajima, K. Igarashi, K. Itou, K. Takeda, and F. Itakura, "Driver identification using driving behavior signals," IEICE - Trans. Inf. Syst., vol. E89-D, no. 3, pp. 1188-1194, 2006.

[11] Y. Takei and Y. Furukawa, "Estimate of driver's fatigue through steering motion." IEEE International Conference on Systems, Man and Cybernetics., vol. 2, p. 1765-1770, 2005.

[12] J. C. McCall, M. M. Trivedi, D. Wipf, and B. Rao, "Lane change intent analysis using robust operators and sparse bayesian learning," in CVPR '05: Proceedings of the 2005 IEEE Computer Society Conference on Computer Vision and Pattern Recognition (CVPR'05) - Workshops. Washington, DC, USA: IEEE Computer Society, 2005, p. 59.

[13] "Volvo car corporation," http://www.media.volvocars.com.

[14] "Mercedes-benz," http://www.emercedesbenz.com/Aug08.

[15] "Lexus," http://www.testdriven.co.uk/lexus-1s-600h

[16] T. D’Orazio, M. Leo, C. Guaragnella, and A. Distante, "A visual approach for driver inattention detection," Pattern Recogn., vol. 40, no. 8, pp. 2341-2355, 2007.

[17] M. Suzuki, N. Yamamoto, O. Yamamoto, T. Nakano, and S. Yamamoto, "Measurement of driver's consciousness by image processing -a method for presuming driver's drowsiness by eye-blinks coping with individual differences." Systems, Man and Cybernetics, vol. 4, p. 2891-2896, 2006.

[18] Q. Ji and X. J. Yang, "Real-time eye, gaze, and face pose tracking for monitoring driver vigilance," Real-Time Imaging, vol. 8, no. 5, pp. 357-377, 2002.

[19] L. Bretzner and M. Krantz, "Towards low-cost systems for measuring visual cues of driver fatigue and inattention in automotive applications," Vehicular Electronics and Safety. IEEE International Conference, pp. 161-164, 2005.

[20] J. Heinzmann, D. Tate, and R. Scott, "Using technology to eliminate drowsy driving," SPE International Conference on Health, Safety, and Environment in Oil and Gas Exploration and Production, April 2008.

[21] "Smart eye,"

http://www.smarteye.se.

[22] "Seeingmachines,"

http://www.seeingmachines.com.

[23] J. Bos, W. Bles, and E. Groen, "A theory on visually induced motion sickness," Displays, vol. 29, no. 2, pp. 47-57, 2008.

[24] T. Åkerstedt, B. Peters, A. Anund, and G. Kecklund, "Impaired alertness and performance driving home from the night shift: a driving simulator study," Journal of Sleep Research, vol. 13, no. 1, pp. 17-20.

[25] "Tutor [online]," http://www.landersimulation.com/index.php.

[26] B. Lang, A. Parkes, S. Cotter, R. Robbins, C. Diels, P. Vanhulle, G. Turi, E. Bekiaris, M. Panou, and J. K. . S. Poschadel, "Train-all integrated system for driver training and assessment using interactive education tools and new training curricula for all modes of road transport," 2007.

[27] N. Stanton, P. Salmon, G. Walter, and C. B. . D. Jenkins, "Human factors methods: A practical guide for engineering and design," 2005.

[28] K. Kaida, M. Takahashi, T. Akerstedt, A. Nakata, Y. Otsuka, and T. H. . K. Fukasawa, "Validation of the karolinska sleepiness scale against performance and eeg variables."

[29] P. Viola and M. J. Jones, "Robust real-time face detection," Int. J. Comput. Vision, vol. 57, no. 2, pp. 137-154, 2004.

[30] J. F. May and C. L. Baldwin, "Driver fatigue: The importance of identifying causal factors of fatigue when considering detection and countermeasure technologies," Transportation Research Part F: Traffic Psychology and Behaviour, vol. 12, no. 3, pp. 218 - 224, 2009. [Online]. Available: http://www.sciencedirect.com/science/article/B6VN84VFBYG1-1/2/07087f8c3b6f88f9e9ed6996388d01ed
[31] A. Kircher, M. Uddman, and J. Sandin, "Vehicle control and drowsiness," vol. 0, 2002.

[32] A. C. Patel and M. K. Markey, "Comparision of three-class classification performance metrics: a case study in breast cancer cad," 2005 , pp. 581-589. 